\title{
Artigos
}

\section{Aspectos médico-legais das substâncias utilizadas como facilitadoras de crime}

\section{Medical-legal aspects of drug-facilitated crime}

\author{
Juliana Takitane ${ }^{1}$, Douglas Sani Pimenta ${ }^{2}$, Fabio Masashi Fukushima ${ }^{3}$, \\ Viviane Gomes da Fonte ${ }^{4}$, Vilma Leyton ${ }^{5}$
}

DOI: http://dx.doi.org/10.11606/issn.2317-2770.v22i2p66-71

\begin{abstract}
Takitane J, Pimenta DS, Fukushima FM, Fonte VG, Leyton V. Aspectos médico-legais das substâncias utilizadas como facilitadoras de crime. Saúde, Ética \& Justiça. 2017;22(2):66-71.

RESUMO: O uso de substâncias modificadoras de comportamento para cometimento de delitos não é um fenômeno recente, elas já foram utilizadas para o cometimento de roubos, homicídios, estupro e violência sexual. A prevalência e o tipo de droga utilizada variam de acordo com o país estudado, mas seu número real ainda é de difícil mensuração frente à hesitação da vítima em contatar as autoridades policiais competentes ou, ainda, ao longo tempo decorrido entre o fato, a denúncia, a coleta de material(is) biológico(s) e a análise deste(s) espécime(s). No Brasil, diferentemente do observado no resto do mundo, os crimes facilitados por drogas têm como finalidade principal o roubo e têm como principais vítimas indivíduos do sexo masculino. São conhecidas mais de 100 substâncias empregadas para estes fins, sendo as principais o etanol, os benzodiazepínicos, o gama-hidroxibutirato (GHB) e a cetamina. Algumas características destas drogas incluem a produção de certos sintomas inespecíficos nas vítimas (desinibição, sedação/hipnose, confusão mental, amnésia anterógrada), além de serem de fácil obtenção, de difícil detecção analítica, permitirem uma administração discreta e início rápido dos efeitos. Diante do exposto e da gravidade dos crimes aos quais tais substâncias estão relacionadas, torna-se evidente a necessidade de investimentos em pesquisa e levantamentos epidemiológicos de qualidade, que possibilitem a geração e a divulgação de informações fidedignas que reflitam o problema.
\end{abstract}

DESCRITORES: Crimes Sexuais; Agentes Psicoativos; Medicina Legal.

\footnotetext{
1. Departamento de Medicina Legal, Ética Médica e Medicina Social e do Trabalho da Faculdade de Medicina da Universidade de São Paulo. Doutoranda em Ciências, área de concentração Fisiopatologia Experimental, da Faculdade de Medicina da Universidade de São Paulo. julianatakitane@gmail.com

2. Departamento de Medicina Legal, Ética Médica e Medicina Social e do Trabalho da Faculdade de Medicina da Universidade de São Paulo. Médico Residente de Medicina Legal do Departamento de Medicina Legal, Ética Médica e Medicina Social e do Trabalho da Faculdade de Medicina da Universidade de São Paulo.dsp_medtau41@hotmail.com

3. Departamento de Medicina Legal, Ética Médica e Medicina Social e do Trabalho da Faculdade de Medicina da Universidade de São Paulo. Médico Residente de Medicina Legal do Departamento de Medicina Legal, Ética Médica e Medicina Social e do Trabalho da Faculdade de Medicina da Universidade de São Paulo. fabio.fukushima@yahoo.com.br

4. Departamento de Medicina Legal, Ética Médica e Medicina Social e do Trabalho da Faculdade de Medicina da Universidade de São Paulo. Médica Residente de Medicina Legal do Departamento de Medicina Legal, Ética Médica e Medicina Social e do Trabalho da Faculdade de Medicina da Universidade de São Paulo. vivizinhagf@hotmail.com

5. Departamento de Medicina Legal, Ética Médica e Medicina Social e do Trabalho da Faculdade de Medicina da Universidade de São Paulo. Professora Doutora do Departamento de Medicina Legal, Ética Médica e Medicina Social e do Trabalho da Faculdade de Medicina da Universidade de São Paulo. vileyton@gmail.com
} 


\section{INTRODUÇÃO}

$\mathbf{O}$ uso de substâncias modificadoras de comportamento para cometimento de delitos não é um fenômeno recente, tendo, essas drogas, sido utilizadas para o cometimento de roubos, homicídios, estupro e violência sexual ${ }^{\mathbf{1 , 2}}$. A prevalência e o tipo de droga utilizada variam de acordo com o país estudado, mas seu número real ainda é de difícil mensuração frente à hesitação da vítima em contatar as autoridades policiais competentes ou, ainda, ao longo tempo decorrido entre o fato, a denúncia, a coleta de material(is) biológico(s) e a análise deste(s) espécime $(s)^{2,3}$.

O principal crime facilitado por uso de drogas no mundo é o estupro ${ }^{1}$. No Brasil, quando não há o necessário discernimento para a prática do ato ou se a vítima não pode oferecer resistência, o crime é enquadrado em estupro de vulnerável, de acordo com o artigo 217-A do Código Penal Brasileiro, o qual apresenta uma penalidade agravada $^{4}$.

De acordo com Anderson et al. ${ }^{5}$, em 2017, o termo drug-facilitated sexual assault (DFSA) refere-se à violência sexual em que a vítima está incapacitada e/ ou incapaz de prover consentimento ao ato sexual como resultado do consumo de álcool ou droga. Gee et al. ${ }^{6}$, em 2006, descreveram duas categorias de DFSA: (a) "DFSA proativa", na qual à vítima é administrada uma substância incapacitante ou desinibidora, secretamente ou de maneira forçada, pelo agressor, com fins de praticar o abuso sexual; e (b) "DFSA oportunista", na qual o agressor se aproveita do estado inebriado da vítima, alcançado pelo consumo voluntário de álcool ou droga pela mesma, para a prática da agressão sexual. Em ambos os casos, a vítima apresenta prejuízos no seu estado de consciência e capacidade reduzida para resistir às ações indesejadas.

Para Hindmarch et al. ${ }^{7}$ ( 2001) o álcool apenas, ou associado a outras drogas é a substância mais comumente encontrada neste tipo de crime, seguido por Cannabis. Este resultado, corroborado por Anderson et al. ${ }^{5}$ (2017) contraria o entendimento comum e sugere que o uso de benzodiazepínicos e hipnóticos não é tão prevalente neste tipo de ocorrência como se pensa. Estes últimos realizaram uma revisão sistemática para encontrar características de violência sexual facilitada por uso de drogas e verificaram que a combinação entre intoxicação alcoólica e uso voluntário de drogas é o maior fator de risco para este tipo de violência sexual, contrariando a crença popular de que é mais comum a indução ao seu uso de forma ardilosa e não consensual ${ }^{5}$.

No Brasil, o termo "Boa Noite, Cinderela" é utilizado popularmente nos casos em que um criminoso faz a vítima ingerir algum tipo de substância que a deixe sem memória para roubar seus pertences. A origem do termo é controversa, porém, segundo relatos, existem duas principais hipóteses. A primeira seria que o nome "Boa Noite, Cinderela" é dado como referência a um programa de televisão brasileira homônimo no qual o apresentador premiava uma participante com uma noite de princesa. A outra hipótese é que a personagem Cinderela é uma princesa que participou de um grande baile, fazendo referência aos locais preferenciais da prática do golpe, como bares e casas noturnas, e que esta perde seu sapato de cristal, como referência à perda do controle pela vítima ${ }^{8}$.

Ao contrário dos países da América Latina e do resto do mundo, no Brasil os crimes facilitados por drogas apresentam um perfil diferente. Em nosso país, esses tipos de crimes têm como finalidade principal o roubo e têm como principais vítimas indivíduos do sexo masculino, tendo ocorrido só na capital paulista 415 roubos deste tipo entre 2014 e a segunda metade de 2017 , segundo mesmo autor'.

Em estudo sobre violência sexual realizado com adolescentes e adultos jovens, foi determinado como principais fatores de risco a precocidade no primeiro encontro e precocidade de início sexual, precocidade da menarca, história anterior de abuso sexual ou vitimização e maior aceitação pessoal de violência contra mulheres ${ }^{\mathbf{1 0}}$.

\section{OBJETIVOS}

Fornecer informações acerca das principais substâncias utilizadas nos crimes facilitados pelo uso de drogas, além dos diferentes tipos de crimes a ele relacionados e o perfil geral das vítimas, tanto no Brasil como em outros países.

\section{MATERIAL E MÉTODOS}

Foi realizada uma revisão de literatura em bases de dados disponíveis na Internet, como Pubmed, Google Scholar, Scielo. A busca por artigos se baseou nos descritores: "drogas facilitadoras de crimes" ("drugfacilitated crimes") e "drogas facilitadoras de abuso sexual" ("drug-facilitated sexual assault").

\section{RESULTADOS E DISCUSSÃO}

Existem mais de 100 Drogas Facilitadores de Crimes (DFCs) listadas nos guias da Sociedade de Toxicologistas Forenses (SOFT) e do Escritório das Nações Unidas sobre Drogas e Crimes (UNODC) ${ }^{11,12}$.

$\mathrm{Na}$ comunidade científica, as substâncias mais conhecidas por serem utilizadas como DFCs são o etanol, os benzodiazepínicos (como por exemplo, o flunitrazepam), o gama-hidroxibutirato (GHB) e a cetamina, podendo ser usadas separadas ou em associação ${ }^{13-15}$.

O GHB já foi uma substância usada por 
fisiculturistas na forma de suplemento alimentar com a finalidade de perder massa gorda, sendo vendido em ginásios e ervanários. No entanto, por ser considerado doping, seu uso para fins esportistas diminuiu, passando a ser consumido em festas tipo "rave", substituindo drogas como o "ecstasy", por exemplo, cujo princípio ativo é a metilenodioximetanfetamina (MDMA), por suas propriedades alucinógenas, euforizantes e hipnóticas sendo, finalmente, usado como DFC, associado ou não com flunitrazepam ${ }^{\mathbf{1 6}, 17}$. Os principais efeitos do GHB são a sensação de euforia e bem-estar, no entanto, os principais efeitos usados para a execução do crime são a amnésia e a incoordenação motora, impedindo a vítima de oferecer resistência e dificultando a memória para lembrar o ocorrido ${ }^{15}$. O efeito inicia-se 20 minutos após a ingestão, dissipando-se após 2 ou 3 horas ${ }^{13}$. A apresentação do GHB é conveniente pela facilidade do transporte e da aplicação, geralmente encontra-se na forma aquosa, também podendo ser encontrado em pó e de fácil dissolução. É de aspecto incolor, inodoro e tem sabor levemente salgado ${ }^{15}$. O GHB é encontrado endogenamente (presente naturalmente no Sistema Nervoso Central dos mamíferos), não sendo detectado por todos os laboratórios e rapidamente eliminado do organismo (totalmente eliminado do sangue em cinco horas e da urina, em 10 horas), o que pode dificultar sua detecção ${ }^{18,19}$. É conhecido como "Ecstasy líquido", "Líquido X”, “G”, "Fantasy”, “Gama-OH”, “Água salgada", "Cherry meth", "Scoop" ou "Easy Lay", podendo ser usado como droga recreativa, também, principalmente entre estudantes e adolescentes ${ }^{20-22}$.

Outra substância bastante utilizada para o cometimento de crimes é o flunitrazepam. Usado na Europa para o tratamento de insônia grave ou em anestesia, o flunitrazepam é um tipo de benzodiazepínico potente ${ }^{23,24}$. Esta substância não tem cheiro, sabor ou cor, sendo facilmente diluída em álcool. O flunitrazepam foi tão utilizado como DFC que o fabricante HoffmanLaRoche alterou o medicamento para um que fosse menos solúvel e que gerasse uma coloração azul, criando uma heterogeneidade ao ser mergulhada em liquido, mudando, inclusive, a disponibilidade para comércio de $2 \mathrm{mg}$ para apenas $1 \mathrm{mg}^{13}$. Devido ao seu uso como DFC, não se comercializa legalmente flunitrazepam no Canadá ou nos EUA, porém, como a venda é facilitada em países como Colômbia e México, tal substância entra ilegalmente naqueles países sob os nomes "Roffies", "Roachies", "Rope" e "Pílula do Esquecer" ("Forget Pill"), sob o facilitador de custar menos de 5 dólares o tablete ${ }^{13,20,25,26}$. Os principais efeitos do flunitrazepam são sedação, redução da ansiedade, desinibição, relaxamento muscular e amnésia ${ }^{25}$. Tais efeitos, principalmente a amnésia, demonstram a conveniência para o criminoso obter o controle desejado da vítima. O início da ação ocorre em 20 minutos após a ingestão, com o pico de efeito em duas horas e duração de até 8 horas $^{13}$. Para o uso como DFC, o flunitrazepam é macerado até virar pó ou dissolvido, mas pode ser fumado, cheirado ou injetado ${ }^{27}$. Pode ser usado como droga recreativa também, inclusive para aumentar o efeito de outras drogas como maconha, cocaína ou álcool ${ }^{27}$. Tem alto potencial em causar dependência química ${ }^{23}$. Nos EUA pode ser encontrado em escolas, clubes ou com gangues. Dos efeitos adversos, o mais importante é a abstinência, que causa cefaleia, dores musculares, ansiedade e inquietude, confusão, alucinações e delírio, convulsões e choque cardiovascular ${ }^{13}$.

A cetamina é composto derivado do cloridrato de fenciclidina (PCP), utilizado como analgésico e anestésico geral na medicina veterinária e em emergências médicas, com restrições ${ }^{28}$. Apesar de seu mecanismo ainda não ter sido completamente desvendado, sabe-se que bloqueia receptores do tipo NMDA e pode interagir com receptores Sigma. A droga produz rapidamente um estado hipnótico, com profunda analgesia, amnésia, não responsividade a comandos, mas o paciente pode manter os olhos abertos, respirar e mover os membros livremente ${ }^{29}$. Apresentase na forma de um pó branco, que pode ser inalado ou misturado com maconha e/ou tabaco e fumado, ou, ainda, um líquido incolor e inodoro, para administração intramuscular. Ambas formas permitem facilmente sua mistura de forma disfarçada às bebidas de potenciais vítimas $^{30}$

Outras drogas usadas como DFCs são maconha, cocaína, barbituratos, opiáceos, triazolam, "Mickey Finn" (mistura entre hidrato de cloral com bebida alcoólica), e outros benzodiazepínicos ${ }^{13}$. Todas essas drogas podem ser usadas como facilitadores de crimes que possuem perfis diferentes, dependendo da localidade em que são cometidos. Um levantamento feito na capital paulista com dados de 414 boletins de ocorrência registrados entre os anos de 2014 e 2017, que citavam o termo "Boa Noite, Cinderela", como é conhecida esta prática no Brasil, mostrou que a maioria das vítimas foram homens $(82,1 \%$, contra $14,0 \%$ mulheres e $3,9 \%$ sem sexo definido). Foi observado também que as vítimas, em sua maioria, eram homossexuais e que os agressores eram geralmente homens atraentes 9 . De acordo com outro levantamento, a finalidade principal deste tipo de abordagem é o roubo, sendo que crimes como roubo, furto e estelionato somaram $95 \%$ dos casos reportados, e o estupro totalizou $5 \%$ dos casos. Segundo a mesma fonte, as principais substâncias utilizadas nestes crimes foram cetamina, GHB e até cocaína ${ }^{31}$. Tais informações, ainda que possam parecer frágeis do ponto de vista científico, foram as únicas publicações encontradas pelos autores que pudessem descrever a realidade brasileira nesse tocante.

De acordo com matéria veiculada no site da British Broadcasting Corporation (BBC), o número de 
crimes envolvendo as "drogas de estupro" tem tido uma evolução muito rápida. A reportagem cita como principais drogas da América Latina os benzodiazepínicos, o GHB, e a burundanga, que tem como princípio ativo o alcaloide escapolamina e que tem caído em desuso nos últimos $\operatorname{anos}^{32}$.

$\mathrm{Na}$ América do Norte, Hindmarch et al. ${ }^{7}$, em 2001, fizeram análise de urina em 3303 amostras, no período entre junho de 1996 e fevereiro de 2000, e encontraram que a droga mais comumente utilizada nos crimes sexuais foi o álcool (sozinho em 44\% dos casos, ou em conjunto com outras substâncias em $23 \%$ ), seguido por Cannabis e medicamentos da classe dos benzodiazepínicos. Entre estes últimos, as drogas mais encontradas foram Oxazepam e Diazepam (71\%). Flunitrazepam foi encontrado em apenas 0,3\% dos casos envolvendo benzodiazepínicos. Além disso, foram detectados cocaína em 14\% dos casos, anfetaminas em $11 \%$ das amostras e GHB em 5\% dos casos.

Em estudo realizado em 2005, no Reino Unido, Scott-Ham e Burton $^{33}$ fizeram análise de sangue e urina de 1014 casos envolvendo a administração de drogas facilitadores de crimes sexuais no reino Unido entre janeiro de 2000 e dezembro de 2002. Álcool foi detectado em $46 \%$ dos casos, drogas ilícitas foram encontradas em $34 \%$, sendo Cannabis a mais frequente (26\%) seguida da cocaína (11\%). Em 21 casos (2\%), uma droga não admitida pela vítima foi encontrada, e pode ter sido administrada sem consentimento, incluindo GHB, diazepam e temazepam.

Hurley et al..$^{34}$ (2006) revisaram casos de alegada violência sexual facilitada por drogas por a partir de dados do Instituto Victoriano de Medicina Forense na Austrália por 12 meses, período concluído em abril de 2003. 76 casos foram selecionados, com histórico de uso de álcool em $77 \%$ destes. $49 \%$ dos participantes disseram usar medicações e $26 \%$ disseram fazer uso de drogas recreativas. Drogas não relatadas pelos participantes foram encontradas em 15 pessoas (20\%). Quatro amostras foram positivas para Cannabis, quatro para antidepressivos, três para anfetaminas, quatro para benzodiazepínicos e três para opiáceos. Este estudo também mostrou que a maioria das vítimas era do sexo feminino (95\%) e com média de idade de 25,6 anos.

O perfil de vítima no Brasil difere quando comparado ao da América Latina e ao do resto do mundo: de acordo com dados encontrados em outros países, a principal motivação dos crimes facilitados por uso de drogas é o estupro². A vítima principal é do sexo feminino, de acordo com um estudo realizado em Paris. Neste, Chèze et al. ${ }^{35}$, em 2005, estudaram 128 casos nesta cidade e reportaram que $85 \%$ das vítimas de crimes facilitados por uso de drogas eram mulheres. Em contrapartida, $82,1 \%$ das vítimas reportadas em levantamento feito no Brasil eram do sexo masculino e $3,9 \%$ não tiveram seu sexo definido 9 . Além disso, outra pesquisa revelou que a natureza jurídica deste tipo de crime no país correspondeu a apenas $5 \%$ dos casos, sendo mais comuns os casos de roubo, furto e estelionato $(87 \%, 23 \% \text { e } 4 \% \text {, respectivamente })^{31}$. Os tipos de drogas encontrados neste tipo de crime no Brasil possuem perfil similar se comparados com as publicações de outros países. Neste país, as principais drogas utilizadas nos crimes facilitados por uso de drogas são álcool, cetamina, flunitrazepam, diazepam e cocaína. Resultados semelhantes são encontrados nos trabalhos de Hurley e colaboradores e no guideline de análise forense de drogas em crimes e violência sexual facilitados por drogas da $\mathrm{ONU}^{12,34}$.

Um ponto importante a ser discutido é a importância do papel das drogas autoadministradas e consentidas nos crimes deste tipo. Para Hindmarch et al. $^{7}$, reforçado pelo trabalho de Anderson et al. ${ }^{\mathbf{5}}$, o chamado "drug spiking", situação em que uma pessoa coloca inadvertidamente uma droga na bebida da vítima, é muito menos comum do que é reportado na mídia. $\mathrm{Na}$ realidade, a grande maioria dos casos envolve grande ingestão de álcool, associada a uso consentido de drogas. O papel das drogas que induzem o efeito de sedação e/ ou amnésia, ainda que importante, não é tão prevalente quanto se imaginava. Deve-se ressaltar, porém, que os dados podem ter sido afetados pela maior dificuldade de detecção de alguns tipos de substâncias, seja pelo tempo de meia-vida mais curto, seja pela limitação da técnica de detecção disponível ou, ainda, simplesmente por não ter sido suspeitado à época da denúncia do crime $e^{5,7,36}$.

\section{CONCLUSÃO}

As drogas mais utilizadas para a prática de crimes são álcool, GHB, benzodiazepínicos, tanto no Brasil quanto no mundo. As vítimas no Brasil são em sua maioria homens, e os criminosos têm como objetivo principal o roubo ou furto. Em outros países, a vítima principal é do sexo feminino e a natureza jurídica do crime é, predominantemente, de crimes sexuais. 
Takitane J et al. Aspectos médico-legais das substâncias utilizadas como facilitadoras de crime.

Takitane J, Pimenta DS, Fukushima FM, Fonte VG, Leyton V. Medical-legal aspects of drug-facilitated crime. Saúde, Ética \& Justiça. 2017;22(2):66-71.

\begin{abstract}
Drug-facilitated crime is not a recent phenomenon and crimes such as robbery, homicide, rape and sexual violence associated with substance consumption have already been reported. The prevalence and class of drugs vary worldwide, but these data are still difficult to measure given the victim's hesitation to contact the competent police authorities or, moreover, due to the extent of time between the fact, the complaint, the collection of biological specimens and their analysis. Unlike in the rest of the world, drug-facilitated crimes in Brazil involve mainly theft and the main victims are men. More than 100 substances are known to be used for this purpose, the main ones being ethanol, benzodiazepines, gammahydroxybutyrate (GHB) and ketamine. Some characteristics of these drugs include the production of certain nonspecific symptoms in the victims (lowered inhibition, sedation / hypnosis, mental confusion, anterograde amnesia), besides being easy to obtain, difficult to detect analytically, allow discreet administration and rapid onset of effects. Given the significance of the crimes related these substances, action is required. Investment in research and quality epidemiological surveys that result in reliable information are urgent.
\end{abstract}

KEY WORDS: Sex Offenses; Psychotropic Drugs; Forensic Medicine.

\title{
REFERÊNCIAS
}

1. Villaim M, Chèze M, Tracqui A, Ludes B, Kintz P. Testing for zolpiclone in hair application to drug-facilitated crimes. Forensic Sci Int. 2004;145(2-3):117-21. DOI: http://dx.doi.org/10.1016/j.forsciint.2004.04.026

2. Shbair MK, Eljabour S, Bassyoni I, Lhermitte M. Drugs involved in drug-facilitated crimes--part II: Drugs of abuse, prescription and over-the-counter medications. A review. Ann Pharm Fr. 2010;68(6):319-31. DOI: http:// dx.doi.org/10.1016/j.pharma.2010.07.003

3. Harper NS. Drug-facilitated sexual assault. In: Jenny C. Child abuse and neglect: diagnosis, treatment and evidence. St Louis (MO): Elsevier Inc.; 2011. p.118-26.

4. Brasil. Presidência da República, Subchefia para Assuntos Jurídicos. Lei $\mathrm{n}^{\mathrm{o}} 12.015$ de 7 de agosto de 2009. Altera o Título VI da Parte Especial do Decreto-Lei no 2.848, de 7 de dezembro de 1940 - Código Penal, e o art. 1ํ da Lei $n^{\circ}$ 8.072, de 25 de julho de 1990, que dispõe sobre os crimes hediondos, nos termos do inciso XLIII do art. 5을 da Constituição Federal e revoga a Lei no 2.252 , de $1^{1}$ - de julho de 1954, que trata de corrupção de menores [Internet]. Brasília, DF; 2009. [Acesso em 2017 fev. 6]. Disponível em: http://www.planalto.gov.br/ccivil_03/ ato2007-2010/2009/lei/112015.htm

5. Anderson LJ, Flynn A, Pilgrim JL. A global epidemiological perspective on the toxicology of drug facilitated sexual assault: A systematic review. J Forensic Leg Med. 2017;47:46-54. DOI: http://dx.doi. org/10.1016/j.jflm.2017.02.005

6. Gee D, Owen P, McLean I, Brentnall K, Thundercloud C. Operation MATISSE: investigating drug facilitated sexual assault. London: The Association of Chief Police Officers (ACPO); 2006.

7. Hindmarch I, ElSohly M, Gambles J, Salamone S. Forensic urinalysis of drug use in cases of alleged sexual assault. J Clin Forensic Med. 2001;8(4):197-205. DOI: http://dx.doi.org/10.1054/jcfm.2001.0513

8. Thais Sant'ana. Por que o golpe é "Boa noite Cinderela", não "Bela Adormecida"? [Internet]. São Paulo; 2016 [Acesso em 2017 fev. 08]. Disponível em: https:// mundoestranho.abril.com.br/cultura/por-que-o-golpe-e- boa-noite-cinderela-nao-bela-adormecida/

9. Marie Declercq. O boa noite Cinderela e o consumo de drogas em São Paulo [Internet]. São Paulo; 2017 [Acesso em 2017 fev. 08]. Disponível em: https://www.vice. com/pt_br/article/ywbynv/o-boa-noite-cinderela-e-oconsumo-de-drogas-em-sao-paulo

10. Rickert VI, Wiemann CM, Vaughan RD, White JW. Rates and risk factors for sexual violence among an ethnically diverse sample of adolescents. Arch Pediatr Adolesc Med. 2004;158(12):1132-9. DOI: http://dx.doi. org/10.1001/archpedi.158.12.1132

11. SOFT - Society of Forensic Toxicologists. Fact Sheet: drug-facilitated sexual assaults [Internet]. Mesa, AZ; 2009 [Acesso em 2017 fev. 08]. Disponível em: http:// www.soft-tox.org/files/DFSA_Fact_Sheet.pdf

12. UNODC - United Nations Office on Drugs and Crime. Guidelines for the forensic analysis of drugs facilitating sexual assault and other criminal acts [Internet]. Nova Iorque; 2011 [Acesso em 2017 fev. 08]. Disponível em: https://www.unodc.org/documents/scientific/forensic analys_of_drugs_facilitating_sexual_assault_and_other_criminal_acts.pdf

13. Jamieson MA. Rohypnol ${ }^{\circledR}$, Gamma Hydroxybutyrate, and drug rape. J Soc Obstet Gynaecol Can. 2001;23(1):38-42. DOI: https://dx.doi.org/10.1016/S0849-5831(16)31095-3

14. Girard LA, Senn YC. The role of the new "Date Rape Drugs" in attributions about date rape. J Interpers Violence. 2008;23(1):3-20.

15. Lima EC, Silva DL. Revisão dos métodos cromatográficos de análise de GHB e análogos. Rev Proc Químicos. 2009;6:17-23.

16. Teter CJ, guthrie SK. A comprehensive review of MDMA and GHB: two common club drugs. Pharmacotherapy. 2001;21(12):1486-513.

17. Guerreiro DF, Carmo AL, Silva JA, Navarro R, Góis C. Club Drugs: um novo perfil de abuso de substâncias em adolescentes e jovens adultos. Acta Med Port. 2011;24:739-56.

18. Lebeau MA, Mozayani A. Drug-facilitated sexual assault: 
Takitane J et al. Aspectos médico-legais das substâncias utilizadas como facilitadoras de crime.

a forensic handbook. $1^{\text {st }}$ ed. San Diego: Academic Press; 2001.

19. Haller C, Thai D, Jacob P, Dyer JE. GHB urine concentrations after single-dose administration in humans. J Anal Toxicol. 2006;30(6):360-4.

20. Smith KM. Drugs used in acquaintance rape. J Amer Pharm Assoc. 1999;39(4):519-25. DOI: https://dx.doi. org/10.1016/S1086-5802(16)30472-7

21. Verjee Z, Giesbrecht E. Abuse of gamma-hydroxybutyrate (liquid ecstacy) with other street drugs. In: Sixth International Congress Of Therapeutic Drug Monitoring And Clinical Toxicology. Cairns, Australia: Lippincott Williams \& Wilkins, Inc; 1999;21(4):470.

22. Slaughter L. Involvement of drugs in sexual assault. J Reprod Med. 2000;45(5):425-30.

23. Hartelius H, Larsson AK, Lepp M, Maim U, Arvidsson A, Dahlström H. A controlled long-term study of flunitrazepam, nitrazepam and placebo, with special regard to withdrawal effects. Acta Psychiatr Scand. 1978;58(1):1-15.

24. Hoffman-LaRoche. Substance Related Rape [Internet] The Vaults of Erowid. Grass Valley, CA; 1999 [Acesso em 2017 fev. 06]. Disponível em: https://erowid.org/ psychoactives/assault/assault_article2.shtml

25. George KA, Dundee JW. Relative amnesic actions of diazepam, flunitrazepam and lorazepam in man. Br J Clin Pharm. 1977;4(1):45-50.

26. Calhoun SR, Wesson DR, Galloway GP, Smith DE. Abuse of flunitrazepam (Rohypnol) and other benzodiazepines in Austin and South Texas. J Psychoactive Drugs. 1996;28(2):183-9. DOI: http://dx.doi.org/10.1080/02791 072.1996 .10524390

27. Bond A, Seijas D, Dawling S, Lader M. Systemic absorption and abuse liability of snorted flunitrazepam. Addiction. 1994;89(7):821-30.

28. Vasconcelos SMM, Andrade MM, Soares PM, Chaves BG, Patrocínio MCA, Sousa FCF, Macêdo DS. Cetamina: aspectos gerais e relação com a esquizofrenia. Rev Psiq Clín. 2005;32(1):10-6. DOI: http://dx.doi.org/10.1590/ S0101-60832005000100002

Recebido para publicação em: 14/09/2017

Aceito para publicação em: 08/11/2017
29. NIH - National Institutes of Health. Drug record: Ketamine [Internet]. Maryland; 2014 [Acesso em 2017 fev. 9]. Disponível em: https://livertox.nlm.nih.gov// Ketamine.htm

30. Adamowicz P, Kala M. Urinary excretion rates of ketamine and norketamine following therapeutic ketamine administration: method and detection window considerations. J Anal Toxicol. 2005;29(5):378-82.

31. Paula Paiva Paulo. Golpe 'boa noite, Cinderela' em SP: maior parte das vítimas é homem e objetivo do crime é roubo [Internet]. São Paulo; 2017 [Acesso em 2017 fev. 07]. Disponível em: https:/g1.globo.com/sao-paulo/ noticia/golpe-boa-noite-cinderela-em-sp-maior-partedas-vitimas-e-homem-e-objetivo-do-crime-e-roubo. ghtml

32. Martin Riepl. Crescente uso de 'drogas do estupro' na América Latina preocupa autoridades [Internet]. São Paulo; 2016 [Acesso em 2017 fev. 06]. Disponível em: http://www.bbc.com/portuguese/internacional-36346967

33. Scott-Ham M, Burton FC. Toxicological findings in cases of alleged drug facilitated sexual assault in the United Kingdom over a three-year period. J Clin Forensic Med. 2005;12(4):175-86. DOI: https://dx.doi.org/10.1016/j. jcfm.2005.03.009

34. Hurley M., Parker H, Wells DL. The epidemiology of drug facilitated sexual assault. J Clin Forensic Med. 2006;13(4):181-5. DOI: http://dx.doi.org/10.1016/ jcfm.2006.02.005

35. Chèze M, Duffort G, Deveaux M, Pépin G. Hair analysis by liquid chromatography-tandem mass spectrometry in toxicological investigation of drug-facilitated crimes: Report of 128 cases over the period June 2003-May 2004 in metropolitan Paris. Forensic Sci Int. 2005;153(1):3-10. DOI: http://dx.doi.org/10.1016/j.forsciint.2005.04.021

36. Dinis-Oliveira RJ, Magalhães T. Forensic toxicology in drug-facilitated sexual assault. Toxicol Mech Methods. 2013;23(7):471-8. DOI: http://dx.doi.org/10.3 $109 / 15376516.2013 .796034$ 